\title{
SIMULTANEOUS DIOPHANTINE APPROXIMATION ON POLYNOMIAL CURVES
}

\author{
NATALIA BUDARINA, DETTA DICKINSON AND JASON LEVESLEY
}

Abstract. The Hausdorff dimension and measure of the set of simultaneously $\psi$-approximable points lying on integer polynomial curves is obtained for sufficiently small error functions.

$\S 1$. Introduction and notation. In dimensions higher than one there are two standard forms of Diophantine approximation and they have rather different properties. To describe these ideas some notation and terminology is needed. For each $t \in \mathbb{R}$ let

$$
\|t\|=\min \{|t-r|: r \in \mathbb{Z}\}=\operatorname{dist}(t, \mathbb{Z}),
$$

and for each $\mathbf{x}=\left(x_{1}, \ldots, x_{k}\right) \in \mathbb{R}^{k}$ let

$$
\|\mathbf{x}\|=\max \left\{\left\|x_{1}\right\|, \ldots,\left\|x_{k}\right\|\right\}
$$

The supremum norm will be denoted by $|$.$| , that is, for a vector \mathbf{x} \in \mathbb{Z}^{n}$,

$$
|\mathbf{x}|=\max \left\{\left|x_{1}\right|, \ldots,\left|x_{n}\right|\right\} \text {. }
$$

Throughout this paper, $\operatorname{hcf}(x, y)$ will be used to denote the highest common factor of the integers $x$ and $y$.

Let $\psi$ be a decreasing function such that $\psi(r) \rightarrow 0$ as $r \rightarrow \infty$. The set $\mathfrak{S}_{\psi}(M)$ of simultaneously $\psi$-approximable points lying on an $m$-dimensional manifold $M$ embedded in $\mathbb{R}^{n}$ is defined by

$$
\mathfrak{S}_{\psi}(M)=\{\mathbf{x} \in M:\|q \mathbf{x}\|<\psi(|q|) \text { for infinitely many } q \in \mathbb{Z}\} .
$$

There is a natural dual to this set, namely

$$
\mathfrak{L}_{\psi}(M)=\left\{\mathbf{x} \in M:\|\mathbf{q} \cdot \mathbf{x}\|<\psi(|\mathbf{q}|) \text { for infinitely many } \mathbf{q} \in \mathbb{Z}^{n}\right\} .
$$

If $\psi(r)=r^{-\tau}$ then the sets are denoted $\mathfrak{S}_{\tau}(M)$ and $\mathfrak{L}_{\tau}(M)$, respectively. Obviously any element of $\mathbb{Q}^{n}$ lying on $M$ is in $\mathfrak{S}_{\psi}(M)$ for all functions $\psi$. Correspondingly, the intersection of $M$ with a rational hyperplane given by the equation $\mathbf{q} \cdot \mathbf{x}=p$ (for $p \in \mathbb{Z}$ and $\mathbf{q} \in \mathbb{Z}^{m}$ ) is contained in $\mathfrak{L}_{\psi}(M)$ for all $\psi$. Any other points in either $\mathfrak{S}_{\psi}(M)$ or $\mathfrak{L}_{\psi}(M)$ lie "close" to these points or planes. In this paper we will study $\mathfrak{S}_{\psi}(M)$ when $M$ is a polynomial curve and $\psi(r) \rightarrow 0$ sufficiently fast. 
Many results in Diophantine approximation are of a metrical nature, that is, they hold on a set of full or zero measure. As embedded manifolds are of measure zero in the ambient space $\mathbb{R}^{n}$, it is appropriate to work with the relative measure induced by the manifold. For any $S \subset M$, the induced Lebesgue measure of $S$ relative to $M$ will be denoted by $|S|$. A manifold $M$ embedded in $\mathbb{R}^{n}$ is said to be extremal if $\left|\mathfrak{S}_{\tau}(M)\right|=0$ for $\tau>1 / n$, or equivalently (using Khintchine's transference principle [7]) if $\left|\mathfrak{L}_{\tau}(M)\right|=0$ for $\tau>n$. Manifolds satisfying various geometric, analytic and number theoretic properties have been shown to be extremal. A general result is due to Kleinbock and Margulis [22] who proved that a non-degenerate manifold is extremal. This has been extended by Kleinbock to a larger class of manifolds in [21]. Non-degeneracy is a generalization of the idea of non-zero curvature and means that for almost all points on the manifold there exists an $l \in \mathbb{N}$ such that the partial derivatives of an appropriate parametrization up to order $l$ span $\mathbb{R}^{n}$. It has been shown $($ see $[3,4, \mathbf{8}])$ that for any non-degenerate manifold $M$ the set $\mathfrak{L}_{\psi}(M)$ satisfies a "zero-one" law. That is, depending on the divergence or convergence of a certain sum, the set has full or zero Lebesgue measure, respectively. (This proves the Baker-Sprindzuk conjecture.)

One would expect that as $\tau$ increases, the size of the sets $\mathfrak{L}_{\tau}$ and $\mathfrak{S}_{\tau}$ should decrease, and this leads naturally to questions concerning Hausdorff dimension in the case of zero Lebesgue measure. It was proved by Baker in [1] that for any planar curve $C$ with non-zero curvature except for a set of Hausdorff dimension zero, the Hausdorff dimension $\operatorname{dim} \mathfrak{L}_{\tau}(C)$ of $\mathfrak{L}_{\tau}(C)$ for $\tau \geq 2$ is

$$
\operatorname{dim} \mathfrak{L}_{\tau}(C)=\frac{3}{\tau+1} .
$$

(When $\tau \leq 2, \mathfrak{L}_{\tau}(C)=C$ by Dirichlet's theorem.) Bernik [6] obtained the Hausdorff dimension $(n+1) /(\tau+1)$ for $\mathfrak{L}_{\tau}(C)$ when $C$ is the Veronese curve (i.e. it is the set of points $\left.\left(x, x^{2}, \ldots, x^{n}\right) \in \mathbb{R}^{n}\right)$. Also, the Hausdorff dimension of $\mathfrak{L}_{\tau}(M)$ was shown to be $m-1+(n+1) /(\tau+1)$ for $m$-dimensional $C^{3}$ manifolds $M$ with $m \geq 2$ on which there are two non-vanishing principal curvatures except on a set of Hausdorff dimension $m-1$ [15]. This dimension is a lower bound when $M$ is extremal and $C^{1}$ [12]. The upper bound is still an open question. On the other hand, very little is known about the set $\mathfrak{S}_{\tau}(M)$ although there does exist a Khintchine type theorem for 2-convex $C^{3}$ manifolds [16] and an asymptotic formula holds under fairly restrictive curvature conditions [17]; for further details see [7]. Recently, the Hausdorff dimension of the set of simultaneously $\tau$-approximable points lying on any planar curve was determined in [5] for $1 / 2 \leq \tau \leq 1$ and shown to be $(2-\tau) /(1+\tau)$. For sufficiently large $\tau$ there also exist results for the unit circle centred at the origin [13], the parabola [2] and quadric surfaces [18]. To investigate this problem further in this paper, a similar result will be obtained for polynomial curves in $\mathbb{R}^{n}$. Unlike Baker's result [1], which holds for all polynomial curves in $\mathbb{R}^{2}$, there is no single formula for the Hausdorff dimension of $\mathfrak{S}_{\tau}(M)$. These results also give a partial answer to Problem 5 in [10], regarding the spectrum of a certain Diophantine exponent. 
§2. Main results. Let

$$
\Gamma=\left\{\left(x, P_{1}(x), \ldots, P_{n-1}(x)\right) \in \mathbb{R}^{n} \mid P_{j} \in \mathbb{Z}[x]\right\}
$$

be a polynomial curve in $\mathbb{R}^{n}$. Let $d_{j}=\operatorname{deg} P_{j}$ and let $d=\max _{j=1, \ldots, n-1} d_{j}$. Note that if $d=1$ then $\Gamma$ is a straight line in $\mathbb{R}^{n}$ defined by integer linear equations. Throughout the paper $\psi$ will be a decreasing function of the positive reals and the function $\phi: \mathbb{R}^{+} \rightarrow \mathbb{R}^{+}$given by $\phi(r)=\psi\left(r^{d}\right) r^{-d}$ will be used for convenience. Also, $f: \mathbb{R}^{+} \rightarrow \mathbb{R}^{+}$will be a dimension function; that is, an increasing continuous function such that $f(r) \rightarrow 0$ as $r \rightarrow 0$. Finally, in what follows, the Vinogradov notation will be used; that is, for $a, b \in \mathbb{R}^{+}, a \ll b$ will mean that $a \leq c b$ for some constant $c>0$. The first theorem in this paper is an analogue of [14, Theorem 1] and concerns the Hausdorff measure of $\mathfrak{S}_{\psi}(\Gamma)$.

THEOREM 1. Let $\psi: \mathbb{R}^{+} \rightarrow \mathbb{R}^{+}$be a decreasing function such that $r^{d-1} \psi(r) \rightarrow 0$. Let $\phi(r)=\psi\left(r^{d}\right) r^{-d}$ and assume that $r^{2} \phi(r) \rightarrow 0$ as $r \rightarrow \infty$ and also that $r^{2} \phi(r)$ is non-increasing. Let $f$ be a dimension function such that $r^{-1} f(r) \rightarrow \infty$ as $r \rightarrow 0$ and $r^{-1} f(r)$ is non-increasing. Furthermore, suppose that $r^{2} f(\phi(r))$ is non-increasing and that for each constant $C>0$ there exists another constant $L>0$ such that $f(\phi(C r)) \leq L f(\phi(r))$ for all sufficiently large $r$. Then the Hausdorff $f$ measure of $\mathfrak{S}_{\psi}(\Gamma)$ satisfies

$$
\mathcal{H}^{f}\left(\mathfrak{S}_{\psi}(\Gamma)\right)= \begin{cases}0 & \text { if } \sum_{r=1}^{\infty} r f(\phi(r))<\infty \\ \infty & \text { if } \sum_{r=1}^{\infty} r f(\phi(r))=\infty\end{cases}
$$

The final condition on $f(\phi(C r))$ is only needed to prove the convergence part, and the other growth conditions on $f$ and $\phi$ are needed in the divergence case. The remaining growth condition on $\psi$ is used to ensure that the approximating rationals are restricted to lying on $\Gamma$.

The next theorem follows from Theorem 1 and provides a partial answer to the question of the Hausdorff dimension of $\mathfrak{S}_{\tau}(\Gamma)$.

THEOREM 2. For $\tau \geq \max (d-1,1)$ the Hausdorff dimension of $\mathfrak{S}_{\tau}(\Gamma)$ is

$$
\operatorname{dim} \mathfrak{S}_{\tau}(\Gamma)=\frac{2}{d(\tau+1)} .
$$

Take $\psi(r)=r^{-\tau}$ and $f(r)=r^{s}$ and this theorem follows directly from Theorem 1. The dimension above will hold as a lower bound for all $\tau>1$ but is not best possible for $\tau<d-1$ when $d>2$.

Theorem 1 will be proved by considering the convergence and divergence cases separately. The convergence case will be proved using covering and counting arguments and the divergence case by adapting the classical set of wellapproximable numbers.

Before the proof a definition of Hausdorff measure and dimension will be given; further details can be found in $[7,19]$. 
Definition 1 (Hausdorff dimension). Let $E$ be a set in $\mathbb{R}^{n}$ and $f$ a dimension function. A $\delta$-cover of $E$ is a countable collection of sets $C_{i}$, each with diameter less than $\delta$, such that $E \subseteq \bigcup_{i=1}^{\infty} C_{i}$. Define

$$
\mathcal{H}_{\delta}^{f}(E)=\inf \sum_{C_{i} \in \mathcal{C}} f\left(\operatorname{diam} C_{i}\right)
$$

where the infimum is taken over all $\delta$-covers of $E$. The Hausdorff outer $f$ measure $\mathcal{H}^{f}(E)$ is $\lim _{\delta \rightarrow 0} \mathcal{H}_{\delta}^{f}(E)$. To define the Hausdorff dimension let $f(r)=r^{s}$ where $s$ is a non-negative real number; then $\operatorname{dim} E$ is defined as

$$
\operatorname{dim} E=\inf \left\{s \mid \mathcal{H}^{f}(E)=0\right\} .
$$

Proof of Theorem 1. In the first lemma it is shown that if $\psi$ decreases sufficiently quickly then the only rational points which need to be considered actually lie on the polynomial curve under consideration. The lemma is proved for both polynomial curves and more general polynomial surfaces as the proof is the same for each.

LEMMA 1. Let $I \subset \mathbb{R}$ be an bounded interval and let $\Gamma$ represent any polynomial curve or surface of the form $\Gamma=\left\{(\mathbf{x}, \mathbf{y}) \in I^{m} \times \mathbb{R}^{n-m} \mid y_{1}=\right.$ $\left.P_{1}(\mathbf{x}), \ldots, y_{n-m}=P_{n-m}(\mathbf{x})\right\}$ where $P_{i} \in \mathbb{Z}[\mathbf{x}]$. Let $d_{i}=\operatorname{deg} P_{i}$ and assume without loss of generality that $d_{1} \leq d_{2} \leq \cdots \leq d_{n-m}=d$. Let $(\mathbf{x}, \mathbf{y}) \in \mathfrak{S}_{\psi}(\Gamma)$. Finally, let $\psi: \mathbb{R}^{+} \rightarrow \mathbb{R}^{+}$satisfy the growth condition $r^{d-1} \psi(r) \rightarrow 0$ as $r \rightarrow \infty$. If

$$
\left|D x_{i}-t_{i}\right|<\psi(D) \quad \text { and } \quad\left|D y_{j}-r_{j}\right|<\psi(D)
$$

for $i=1, \ldots, m, j=1, \ldots, n-m, D$ a sufficiently large integer and $t_{i}$, $r_{j} \in \mathbb{Z}$, then the point $\left(t_{1} / D, \ldots, t_{m} / D, r_{1} / D, \ldots, r_{n-m} / D\right)$ lies on $\Gamma$.

Proof. Let $(\mathbf{x}, \mathbf{y}) \in \Gamma$ so that $y_{j}=P_{j}(\mathbf{x}), j=1, \ldots, n-m$. Assume that

$$
\begin{gathered}
\left|x_{i}-t_{i} / D\right|<\psi(D) D^{-1} \quad \text { for } i=1, \ldots, m \\
\left|y_{j}-r_{j} / D\right|<\psi(D) D^{-1} \text { for } j=1, \ldots, n-m .
\end{gathered}
$$

Hence, $x_{i}-t_{i} / D=\varepsilon_{i}$ and $y_{j}-r_{j} / D=\eta_{j}$ for some $\varepsilon_{i}$ and $\eta_{j}$ with $\left|\varepsilon_{i}\right|,\left|\eta_{j}\right|<$ $\psi(D) D^{-1}$. Let $\boldsymbol{\varepsilon}=\left(\varepsilon_{1}, \ldots, \varepsilon_{m}\right)$. As

$$
y_{j}=P_{j}(\mathbf{x})=P_{j}(\mathbf{t} / D+\boldsymbol{\varepsilon}) \text { for } j=1, \ldots, n-m
$$

it follows that

$$
\frac{r_{j}}{D}+\eta_{j}=P_{j}\left(\frac{\mathbf{t}}{D}\right)+R_{j}(\varepsilon)
$$

where $R_{j}(\varepsilon)$ is the sum of the remaining terms. Note that $R_{j}(\varepsilon) \ll|\varepsilon|$. Multiplying through by $D^{d_{j}}$, where $d_{j}$ is the degree of $P_{j}$, gives

$$
r_{j} D^{d_{j}-1}-D^{d_{j}} P_{j}(\mathbf{t} / D)=D^{d_{j}}\left(R_{j}(\boldsymbol{\varepsilon})-\eta_{j}\right)
$$

so that

$$
\left|r_{j} D^{d_{j}-1}-D^{d_{j}} P_{j}(\mathbf{t} / D)\right| \ll D^{d_{j}-1} \psi(D)
$$

and the left-hand side is an integer. Due to the growth restrictions on $\psi$, for sufficiently large $D$, the right-hand side is less than one, which implies that the 
left-hand side must equal zero. Therefore

$$
\frac{r_{j}}{D}=P_{j}\left(\frac{\mathbf{t}}{D}\right)
$$

and the point $(\mathbf{t} / D, \mathbf{r} / D)$ lies on $\Gamma$ if $r^{d-1} \psi(r) \rightarrow 0$ as $r \rightarrow \infty$.

If $(x, \mathbf{y}) \in \mathfrak{S}_{\psi}(\Gamma) \backslash \mathbb{Q}^{n}$ then $(x, \mathbf{y})$ is within a distance $\psi(D) D^{-1}$ of a rational point $(p / D, \mathbf{r} / D)$ infinitely often. Furthermore, there are infinitely many distinct rational points satisfying this condition. Hence, by cancelling factors common to all $p_{i}, r_{j}$ and $D,(x, \mathbf{y})$ is within a distance $\psi(D) D^{-1}$ of infinitely many rational points $(p / D, \mathbf{r} / D)$ for which $\operatorname{hcf}\left(p, r_{1}, \ldots, r_{n-1}, D\right)=1$.

To prove the theorem it is more convenient to work with the sets

$$
V_{\psi}(\Gamma)=\left\{x \in I \mid(x, \mathbf{y}) \in \mathfrak{S}_{\psi}(\Gamma)\right\}
$$

where $I \subset \mathbb{R}$ is a bounded interval. Clearly if a set of Hausdorff measure zero or infinity is transformed under a bi-Lipschitz transformation then the Hausdorff measure of the transformed set will also be zero or infinity, respectively. Given that we are dealing with polynomial curves it is not difficult to show via the mean value theorem that if $\mathbf{x}_{1}, \mathbf{x}_{2} \in \Gamma$ then there exists a constant $K>1$ such that

$$
\left|x_{1}^{1}-x_{2}^{1}\right| \leq\left|\mathbf{x}_{1}-\mathbf{x}_{2}\right| \leq K\left|x_{1}^{1}-x_{2}^{1}\right|
$$

where $x_{i}^{1}$ is the first entry in the vector $\mathbf{x}_{i}=\left(x_{i}^{1}, \ldots, x_{i}^{n}\right) \in \Gamma$. Also, since the real line can be written as a countable union of intervals $I$, the Hausdorff measure of $\mathfrak{S}_{\psi}(\Gamma)$ for any curve $\Gamma$ defined on the whole real line can be found by proving that the Hausdorff measure of $V_{\psi}(\Gamma)$ is zero or infinity for a general interval $I$. Hence, to obtain the Hausdorff measure of $\mathfrak{S}_{\psi}(\Gamma)$ it is sufficient to determine the Hausdorff measure of $V_{\psi}(\Gamma)$.

Note that $V_{\psi}(\Gamma)$ can be written as a limsup set in the following way. Let $P_{p, q}=p / q$ with $p \in \mathbb{Z}$ and $q \in \mathbb{N}$ and $\operatorname{hcf}(p, q)=1$. Then the points

$$
P_{p, q}^{\prime}=\left(\frac{p}{q}, \frac{Q_{1}(p, q)}{q^{d_{1}}}, \ldots, \frac{Q_{n-1}(p, q)}{q^{d_{n-1}}}\right)
$$

lie on $\Gamma$; here $Q_{j}(p, q)=q^{d_{j}} P_{j}(p / q) \in \mathbb{Z}$. Let $D$ be the lowest common denominator of the point $P_{p, q}^{\prime}$; that is, there exist integers $t, r_{1}, \ldots, r_{n-1}$ such that the point $P_{p, q}^{\prime}=\left(t / D, r_{1} / D, \ldots, r_{n-1} / D\right)$ lies on $\Gamma$. The standard notation $B(P, r)$ will be used for a ball centred at a point $P$ of radius $r$. As $V_{\psi}(\Gamma)$ is the set of points in $I$ such that the inequalities $|x-t / D|<\psi(D) D^{-1}$ and $\left|y_{j}-r_{j} / D\right|<\psi(D) D^{-1}$ are satisfied infinitely often for $(x, \mathbf{y}) \in \Gamma$ and $j=1,2, \ldots, n-1$ it is readily verified that

$$
\begin{aligned}
& \bigcap_{N=1}^{\infty} \bigcup_{q>N} \bigcup_{0<p<q ;(p, q)=1} B\left(P_{p, q}, \frac{\psi(D)}{K D}\right) \\
& \quad \subseteq V_{\psi}(\Gamma) \subseteq \bigcap_{N=1}^{\infty} \bigcup_{q>N} \bigcup_{0<p<q ;(p, q)=1} B\left(P_{p, q}, \frac{\psi(D)}{D}\right) .
\end{aligned}
$$


Convergence and divergence results for the Hausdorff measure of $V_{\psi}(\Gamma)$ will now be determined.

Lemma 2. Let $\psi: \mathbb{R}^{+} \rightarrow \mathbb{R}^{+}$be a decreasing function with $\phi(r)=$ $\psi\left(r^{d}\right) r^{-d}$ and $f$ a dimension function. Assume that for each constant $C>0$ there exists a constant $L>0$ such that these functions satisfy the growth condition $f(\phi(C r)) \leq L f(\phi(r))$ for all $r$ sufficiently large. If $\sum_{r=1}^{\infty} r f(\phi(r))<\infty$ then

$$
\mathcal{H}^{f}\left(V_{\psi}(\Gamma)\right)=0 .
$$

Proof. A cover for the set will be found. To this end, intervals will be drawn around the approximating points $P_{p, q}$ with side length $\psi(D) D^{-1}$. An estimate for $D$ is needed. It should be clear that $q \leq D \leq q^{d}$. Let $H=\max _{i, j}\left|a_{i}^{j}\right|$ where $a_{i}^{j}$ is the $i$ th coefficient of the polynomial $P_{j}$ in the definition of $\Gamma$.

We may assume without loss of generality that $d=\max _{i} \operatorname{deg} P_{i}=\operatorname{deg} P_{n-1}$. Let $h_{1}=\operatorname{hcf}\left(a_{d}^{n-1}, q\right)$ so that $a_{d}^{n-1}=h_{1} k_{1}$ and $q=h_{1} u_{1}$. Then

$$
\begin{aligned}
P_{n-1}\left(\frac{p}{q}\right) & =\left(\frac{a_{d}^{n-1} p^{d}+a_{d-1}^{n-1} p^{d-1} q+\cdots+a_{0}^{n-1} q^{d}}{q^{d}}\right) \\
& =\left(\frac{\left(k_{1} p^{d}+a_{d-1}^{n-1} p^{d-1} u_{1}\right)+q\left(a_{d-2}^{n-1} p^{d-2} u_{1}+\cdots+a_{0}^{n-1} u_{1} q^{d-2}\right)}{u_{1} q^{d-1}}\right) .
\end{aligned}
$$

Clearly, $\operatorname{hcf}\left(k_{1}, u_{1}\right)=1$ so that $\operatorname{hcf}\left(k_{1} p^{d}+a_{d-1}^{n-1} p^{d-1} u_{1}, u_{1}\right)=1$. Thus, if $h_{2}=\operatorname{hcf}\left(k_{1} p^{d}+a_{d-1}^{n-1} p^{d-1} u_{1}, q\right)$ then $h_{2} \mid h_{1}$. This process can be continued, each time constructing the numbers $h_{i}, k_{i}$ and $u_{i}$ and each time showing that $h_{i} \mid h_{i-1}$ and therefore that $h_{i} \mid h_{1}$. Hence, as $h_{1} \leq a_{d}^{n-1}<H$ we have $D>$ $q^{d} / H^{d}$.

For each $N>0$ we have a cover of $V_{\psi}(\Gamma)$ by intervals $B\left(P_{p, q}, \psi(D) D^{-1}\right)$ such that

$$
V_{\psi}(\Gamma) \subset \bigcup_{q>N} \bigcup_{p \leq q} B\left(P_{p, q}, \psi(D) D^{-1}\right) .
$$

Hence,

$$
\begin{aligned}
\mathcal{H}^{f}\left(V_{\psi}(\Gamma)\right) & \ll \sum_{q>N} \sum_{p \leq q} f\left(\psi(D) D^{-1}\right) \ll \sum_{q>N} q f\left(\psi(D) D^{-1}\right) \\
& \ll \sum_{q>N} q f(\phi(q / H)) \ll \sum_{q>N} q f(\phi(q))<\infty .
\end{aligned}
$$

This follows from the growth condition on $f$ and $\phi$. Therefore, $\mathcal{H}^{f}\left(V_{\psi}(\Gamma)\right)$ $=0$.

For larger error functions $\psi$ there are other rational points lying close to $\Gamma$ but not actually on it which can affect the dimension and measure and must therefore also be considered. In that case, the above convergence argument is no longer valid and the question remains open. 
We now turn to the divergence case. First, note that the points

$$
\left(\frac{p q^{d-1}}{q^{d}}, \frac{Q_{1}(p, q) q^{d-d_{1}}}{q^{d}}, \ldots, \frac{Q_{n-1}(p, q)}{q^{d}}\right)
$$

lie on $\Gamma$ with $Q_{j}(p, q)=q^{d_{j}} P_{j}(p / q)$. Here, again, we are assuming without loss of generality that $d=\max _{i=1, \ldots, n-1} d_{j}=d_{n-1}$.

Let

$$
\tilde{W}(\phi)=\left\{x \in \mathbb{R} \mid \operatorname{dist}\left(x, P_{p, q}\right) \leq \phi(q) / K \text { infinitely often }\right\}
$$

This is a slight variation of the classical set of $\phi$-approximable points in Diophantine approximation on the real line. Clearly $\tilde{W}(\phi) \subseteq V_{\psi}(\Gamma)$. The following theorem with $f(r)=r^{s}$ was proved in [20, Theorem 3] and a higherdimensional version for general $f$ was proved in [14, Theorem 1].

THEOREM 3 (Jarník). Let $f$ be a dimension function such that $r^{-1} f(r) \rightarrow$ $\infty$ as $r \rightarrow 0$ and $r^{-1} f(r)$ is non-increasing. Let $\phi: \mathbb{R}^{+} \rightarrow \mathbb{R}^{+}$be a decreasing function such that $r^{2} \phi(r) \rightarrow 0$ as $r \rightarrow \infty$ and $r^{2} \phi(r)$ is non-increasing. Furthermore, suppose that $r^{2} f(\phi(r))$ is non-increasing. If

$$
\sum_{r=1}^{\infty} f(\phi(r)) r=\infty
$$

then

$$
\mathcal{H}^{f}(W(\phi))=\infty .
$$

It is clear that the conditions on $f$ and $\phi$ are satisfied and that the sum diverges. Hence,

$$
\infty=\mathcal{H}^{f}(\tilde{W}(\phi)) \leq \mathcal{H}^{f}\left(V_{\psi}(\Gamma)\right) .
$$

Therefore, $\mathcal{H}^{f}\left(\mathfrak{S}_{\psi}(\Gamma)\right)=\mathcal{H}^{f}\left(V_{\psi}(\Gamma)\right)=\infty$. This, together with Lemma 2, proves Theorem 1.

§3. Endnotes and examples. As examples of Theorem 2 consider the following curves and surfaces:

$\left(C_{1}\right)\left\{\left(x, x^{3}\right) \in \mathbb{R}^{2}\right\}$;

$\left(C_{2}\right)\left\{\left(x, x^{2}, x^{3}\right) \in \mathbb{R}^{3}\right\}$

$\left(C_{3}\right) \quad\left\{\left(x, x, x^{3}\right) \in \mathbb{R}^{3}\right\}$.

From Theorem $2, \operatorname{dim} \mathfrak{S}_{\tau}\left(C_{i}\right)=2 /(3(\tau+1))$ for $\tau>2$ and $i=1,2,3$. This leaves the question of what happens when $\tau \leq 2$. Since $\left(C_{1}\right)$ is a planar curve it is known from [5] that $\operatorname{dim} \mathfrak{S}_{\tau}\left(C_{1}\right)=(2-\tau) /(1+\tau)$ for $1 / 2 \leq \tau \leq 1-$ what happens for $1 \leq \tau \leq 2$ is as yet unknown. Now $\left(C_{2}\right)$ is extremal and therefore $\mathfrak{S}_{\tau}\left(C_{2}\right)$ certainly has zero measure for $\tau>1 / 3$. On the other hand, $\left(C_{3}\right)$ is not extremal and it can easily be shown from Dirichlet's theorem that $\mathfrak{S}_{\tau}\left(C_{3}\right)$ has full measure for $\tau \leq 1 / 2$ and from Khintchine's transference principle that it has zero measure for $\tau>1 / 2$. In both cases finer results regarding the Hausdorff 
dimension have not been obtained for the remaining ranges and are the subject of ongoing research. In the dual case the situation is very different. From Baker's result [1] $\operatorname{dim} \mathfrak{L}_{\tau}\left(C_{1}\right)=3 /(\tau+1)$ for $\tau>2$; also $\operatorname{dim} \mathfrak{L}_{\tau}\left(C_{2}\right)=4 /(\tau+1)$ for $\tau>3$ was proved by Bernik [6]; and finally $\mathfrak{L}_{\tau}\left(C_{3}\right)=C_{3}$ as it is contained in a rational hyperplane.

There are clearly many unanswered questions, including, for example, what happens for error functions $\psi$ which decrease more slowly; or equivalently, what happens when $\tau$ lies in the middle ranges? What are the approximation properties of non-integral polynomial curves (which need have no rational points lying on them) and more generally any curve or more general polynomial surface? Some small results exist for translated polynomial curves [11] and for some very particular polynomial surfaces [9], but they are by no means best possible.

Acknowledgements. We would like to thank Maurice Dodson for his help and encouragement, and Victor Beresnevich for pointing out some mistakes and for some very helpful comments. N. Budarina is supported by the Science Foundation Ireland grants RFP05/MAT00015 and RFP08/MTH1512.

\section{References}

1. R. C. Baker, Dirichlet's theorem on Diophantine approximation. Math. Proc. Cambridge Philos. Soc. 83 (1978), 37-59.

2. V. Beresnevich, Distribution of rational points near a parabola. Dokl. Nats. Akad. Nauk Belarusi 45 (2001), 21-23 (in Russian).

3. V. V. Beresnevich, A Groshev type theorem for convergence on manifolds. Acta Math. Hungar. 94 (2002), 99-130.

4. V. Beresnevich, V. Bernik, D. Kleinbock and G. Margulis, Metric Diophantine approximation, the Khintchine-Groshev theorem for non-degenerate manifolds. Mosc. Math. J. 2(2) (2002), 203-225.

5. V. Beresnevich, D. Dickinson and S. Velani, Diophantine approximation on planar curves and the distribution of rational points. Ann. of Math. (2) 166(2) (2007), 367-426.

6. V. I. Bernik, An application of Hausdorff dimension in the theory of Diophantine approximation. Acta Arith. 42 (1983), 219-253 (in Russian).

7. V. I. Bernik and M. M. Dodson, Metric Diophantine Approximation on Manifolds and Hausdorff Dimension (Cambridge Tracts in Mathematics 137), Cambridge University Press (Cambridge, 1999).

8. V. Bernik, D. Kleinbock and G. A. Margulis, Khintchine-type theorems on manifolds: the convergence case for standard and multiplicative versions. Int. Math. Res. Not. 9 (2001), 453-486.

9. N. Budarina and D. Dickinson, Simultaneous Diophantine approximation on surfaces defined by simple polynomial expressions. Math. Proc. R. Ir. Acad. (2009) (submitted).

10. Y. Bugeaud and M. Laurent, Exponents of Diophantine approximation. In Diophantine Geometry (Centro di Ricerca Matematica Ennio De Giorgi (CRM) Series 4) (2007) 101-121.

11. D. Dickinson, Ideas and results from the theory of Diophantine approximation. Conference Proceedings: Diophantine Phenomena in Differential Equations and Dynamical Systems (RIMS Kyoto 2004).

12. H. Dickinson and M. M. Dodson, Extremal manifolds and Hausdorff dimension. Duke Math. J. 101(2) (2000), 271-281.

13. H. Dickinson and M. M. Dodson, Simultaneous Diophantine approximation on the circle and Hausdorff dimension. Math. Proc. Cambridge Philos. Soc. 130 (2001), 515-522.

14. D. Dickinson and S. Velani, Hausdorff measure and linear forms. J. Reine Angew. Math. 490 (1997), $1-36$.

15. M. M. Dodson, B. P. Rynne and J. A. G. Vickers, Metric Diophantine approximation and Hausdorff dimension on manifolds. Math. Proc. Cambridge Philos. Soc. 105 (1989), 547-558.

16. M. M. Dodson, B. P. Rynne and J. A. G. Vickers, Khintchine-type theorems on manifolds. Acta Arith. 57 (1991), 115-130. 
17. M. M. Dodson, B. P. Rynne and J. A. G. Vickers, Simultaneous Diophantine approximation and asymptotic formulae on manifolds. J. Number Theory 58 (1996), 298-316.

18. C. Drutu, Diophantine approximation on rational quadrics. Math. Ann. 333 (2005), 405-469.

19. K. Falconer, Fractal Geometry, Wiley (New York, 1989).

20. V. Jarník, Über die simultanen Diophantischen Aproximationen. Math. Z. 33 (1931), 505-543.

21. D. Y. Kleinbock, Extremal subspaces and their sub-manifolds. Geom. Funct. Anal. 13(2) (2003), 437-466.

22. D. Y. Kleinbock and G. A. Margulis, Flows on homogeneous spaces and Diophantine approximation on manifolds. Ann. of Math. (2) 148(2) (1998), 339-360.

Natalia Budarina,

Department of Mathematics,

Logic House,

NUI Maynooth, Co Kildare,

Republic of Ireland

Jason Levesley,

Department of Mathematics,

University of York,

Heslington, York YO10 5DD,

U.K.
Detta Dickinson,

Department of Mathematics,

Logic House,

NUI Maynooth, Co Kildare,

Republic of Ireland

E-mail: ddickinson@maths.nuim.ie 